\title{
Agricultura familiar para el desarrollo rural incluyente
}

\author{
Family agriculture for inclusive rural development \\ Laksmi Reddiar Krishnamurthy ${ }^{1 \ddagger}$, Sumithra Krishnamurthy ${ }^{2}$,
Indumathi Rajagopal ${ }^{1}$ y Arturo Peralta Solares ${ }^{1}$
}

${ }^{1}$ Centro de Agroforestería para el Desarrollo Sostenible de la Universidad Autónoma Chapingo. 56230 Chapingo, Estado de México, México.
${ }^{\ddagger}$ Autor responsable (uach1854@gmail.com)
${ }^{2}$ University of Sussex. Sussex House, Falmer Brighton, BN1 9RH. United Kingdom.

\section{RESUMEN}

Las comunidades indígenas Mayas de la península de Yucatán han practicado la antigua tradición de agricultura familiar, en particular los huertos caseros, para garantizar su seguridad alimentaria. Con el objetivo de mejorar la práctica tradicional considerando paradigmas de la ciencia moderna, por una parte, se colectaron datos para definir la complejidad estructural y diversidad funcional a partir de 20 huertos familiares en cinco comunidades: $X$ - Maben, $X$ - Pichil, $X$ Yatil, San José II y Melchor Ocampo; y por otra, se organizaron grupos de discusión para dilucidar la estrategia de gestión practicada por las comunidades nativas. Los resultados mostraron que los huertos son manejados principalmente por las mujeres. También mostraron que el propósito principal del crecimiento y mantenimiento de los huertos familiares es garantizar la producción de alimentos nutritivos durante todo el año. Y, por último que los huertos caseros también sirven para propósitos secundarios tales como la provisión de productos y servicios para la medicina tradicional. El estudio sugiere que se debe de promover e invertir en huertos caseros para mejorar las estrategias de desarrollo incluyente en ambientes socio-culturales y biofísicos similares.

Palabras clave: seguridad alimentaria, huertos familiares, diversidad de especies, desarrollo sostenible.

\section{SUMMARY}

The indigenous Mayan populations of the Yucatan peninsula of Mexico have practiced the ancient traditions of family farming, especially home gardens, to ensure their food security. With the objective of

Cita recomendada:

Krishnamurthy, L. R., S. Krishnamurthy, I. Rajagopal y A. Peralta Solares. 2017. Agricultura familiar para el desarrollo rural incluyente. Terra Latinoamericana 35: 135-147. improving the traditional practice with modern science, data were collected on the structural complexity and functional diversity from 20 home gardens selected at random in each of the following five communities: X-Maben, X-Pichil, X-Yatil, San José II and Melchor Ocampo. In addition, group discussions were organized to elucidate the management strategy practiced by the indigenous people. The results show that home gardens are managed mainly by women. The main purpose of growing and maintaining home gardens is to guarantee the production of nutritious food all year round. Finally, the home gardens also serve secondary purposes such as provision of products and services for traditional medicine. This study suggests that home gardens should be promoted and invested in to improve inclusive development strategies for contexts with similar socio-cultural and biophysical circumstances.

Index words: food security, home gardens, species diversity, sustainable development.

\section{INTRODUCCIÓN}

Los huertos familiares encarnan una práctica antigua y común de grupos de poblaciones indígenas en todo el mundo (Eyzaguirre y Linares, 2004). Los huertos familiares, generalmente integran múltiples cultivos y sirven para varios propósitos (Galhena et al., 2013), los cuales incluyen la seguridad alimentaria y económica, pero también el intercambio de conocimientos y la cohesión de la comunidad. Aunque existen varias definiciones de "huertos familiares" (Torquebiau, 1992; Méndez et al., 2011), para efectos del presente, los huertos familiares se consideran sistemas de agricultura que combinan diferentes funciones físicas, sociales y económicas en el área del solar alrededor de la casa familiar.

Recibido: junio de 2016. Aceptado: noviembre de 2016. Publicado en Terra Latinoamericana 35: 135-147. 
La práctica de los huertos familiares se considera una de las actividades más antiguas de uso del suelo; ha evolucionado a través de generaciones con una progresiva intensificación del uso del suelo (Kumar y Nair, 2004). El concepto de la base operativa de los huertos familiares esta en las estrechas combinaciones de árboles, arbustos y cultivos anuales, a veces asociados con animales domésticos alrededor del hogar (Wiersum, 1982; Brownrigg, 1985; Fernandes y Nair, 1986; Soemarwoto, 1987; Kumar y Nair, 2004). Los huertos familiares, aunque practicados en diferentes sectores socioeconómicos, son predominantemente adoptados por los agricultores de subsistencia y están generalizados, principalmente en climas tropicales en entornos rurales (Galhena et al., 2013), por lo general son administrados por la madre de familia (Caballero, 1992). Debido a estas consideraciones, los huertos caseros han representado una dimensión sobre el tipo de acciones que representan el desarrollo incluyente; al cual se le puede entender como una serie de actividades en las que se reduce la pobreza al mismo tiempo que los distintos grupos de cada sociedad se ven involucrados en el proceso de toma de decisiones y de participación. (PNUD, 2016). Una característica distintiva de los huertos familiares es la presencia de una gran diversidad de especies de diferentes grupos funcionales tales como las hortalizas, árboles frutales, plantas medicinales, especias y condimentos, bebidas, plantas ornamentales, así como animales domésticos y salvajes.

Varios estudios sobre huertos familiares se han centrado en su complejidad estructural (Soemarwoto, 1987; Arias-Reyes, 2012; Flores-Guido, 2012; Mariaca, 2012), función (Fernandes y Nair, 1986), biodiversidad, seguridad alimentaria y gestión de nutrientes (Montagnini, 2006; Cahuich-Campos, 2012), ganancias económicas (Mohan et al., 2006, Cámara-Cordova, 2012), y cuestiones de sostenibilidad (Torquebiau, 1992; Torquebiau y Penot, 2006). A pesar de recibir altas calificaciones en las funciones productivas y de servicios, a los huertos familiares no se les ha dado importancia como estrategia de desarrollo incluyente. Los huertos familiares han pasado a primer plano como meras prácticas para garantizar la seguridad alimentaria en las zonas y comunidades marginales.

Los huertos familiares cumplen un papel crucial para garantizar la seguridad alimentaria de los hogares en poblaciones indígenas. A nivel mundial, los huertos familiares tienen una importancia estratégica para el cumplimiento de los Objetivos de Desarrollo del Milenio y los subsecuentes Objetivos de Desarrollo Sostenible, donde se plantean como una prioridad acabar con la pobreza alimentaria y crear un exitoso acceso a alimentos nutritivos. La seguridad alimentaria y la soberanía alimentaria se pueden entender como dos partes complementarias de un objetivo común. La seguridad alimentaria, por un lado se refiere a la condición en donde "en todo momento, todas las personas tienen acceso físico, social y económico a alimentos suficientes, seguros y nutritivos que pueden cumplir con sus necesidades dietéticas y preferencias alimenticias para una vida activa y sana" (FAO, 1996). La soberanía alimentaria por otro lado, es el derecho al acceso a alimentos sanos y culturalmente apropiados y producidos a través de métodos ecológicamente sensibles y sostenibles, así como el derecho a definir los sistemas de agricultura y de alimentos de uno mismo (Vía Campesina, 1996).

En este contexto, la incorporación de la perspectiva de la seguridad alimentaria en la agenda internacional de desarrollo ha tenido un efecto transformador en la investigación de los huertos familiares, especialmente desde el año 2000 cuando se acordaron los Objetivos de Desarrollo del Milenio, donde la investigación sobre huertos familiares se ha orientado hacia la inclusión de los temas de sostenibilidad y resiliencia. La resiliencia, la capacidad de un sistema a sobreponerse al cambio social, político y ambiental se logra mediante la disponibilidad de fuentes de alimentos e ingresos adicionales fuera del empleo tradicional (FAO, 1996). La sostenibilidad, la calidad de una práctica que no es perjudicial para el medio ambiente, tanto socialmente como ecológicamente (United Nations, 2000; FAO, 2013), es también un componente clave de las prácticas de los huertos familiares puesto que su huella ecológica es normalmente muy baja (Galhena et al., 2013).

El reporte de CONEVAL (2013), estima que el $25 \%$ de la población de México vive en condiciones de pobreza alimentaria; por lo que el Gobierno puso en marcha la Cruzada Contra el Hambre que tiene como objetivo lograr la seguridad alimentaria nacional, manteniendo la sostenibilidad del medio ambiente a la vez. En este contexto los huertos familiares pueden jugar un papel significativo, ya que pueden facilitar resultados de seguridad alimentaria sin poner en peligro las condiciones ambientales. 
En México, y a fin de comprender la incorporación de los huertos familiares, es importante tener en cuenta el papel que los huertos familiares han desempeñado históricamente. De hecho, los huertos familiares desempeñaron un papel importante para las sociedades pre-coloniales, como los mayas, aztecas y totonacas (Caballero, 1992). A través de estos sistemas, las poblaciones fueron capaces de desarrollar asentamientos con una producción anual sostenida de alimentos (Angel-Pérez, 2013). Por otra parte, las comunidades fueron capaces de establecer relaciones con las comunidades cercanas por medio del comercio, y estas prácticas continuaron durante y después de la época colonial (Caballero, 1992). En este sentido, los huertos familiares han permitido la creación de condiciones de vida a través de la resiliencia y la seguridad alimentaria, económica y social. En la actualidad, la combinación de estos aspectos por medio de huertos familiares representa una forma de desarrollo incluyente como practica por la cual se incluye equitativamente a la población más vulnerable en los procesos de desarrollo nacional e internacional (UNEP, 2012), mismos que son practicados ampliamente en algunas de las zonas más pobres de México (Rebollar-Domínguez et al., 2008).

El presente estudio examina: 1) el papel de los huertos familiares para el desarrollo rural, con un enfoque específico en la contribución a la seguridad alimentaria en el contexto de las poblaciones indígenas mayas de la península de Yucatán, México y 2) la complejidad estructural, diversidad funcional y la estrategia de gestión de los huertos familiares mayas. Así como el papel que pudieran desempeñar los huertos familiares como estrategia viable para el desarrollo incluyente, especialmente en las zonas marginales que quedan fuera de los beneficios de las tecnologías de producción promovidas durante las últimas décadas.

\section{MATERIALES Y MÉTODOS}

\section{Sitio de Estudio}

La investigación se llevó a cabo en el municipio de Felipe Carrillo Puerto en el estado de Quintana Roo, en el sureste de México (19०03' y $20^{\circ} 25^{\prime} \mathrm{N}$; $87^{\circ} 25^{\prime}$ y $88^{\circ} 43^{\prime} \mathrm{O}$; altitud de $0-100 \mathrm{~m}$; precipitación anual de $1250 \mathrm{~mm}$; clima cálido (tropical seco Köppen: DBCity, 2016) y húmedo con suelos Leptosoles réndzico en mayor predominancia (Bautista et al., 2005). La población de la zona es de ascendencia maya, dentro de su actividad incluyen la práctica de huertos familiares. En el estado de Quintana Roo, el 44.4\% de la población es indígena (INEGI, 2015) y el $95 \%$ son maya parlantes (INEGI, 2016).

\section{Muestreo y Recolección de Datos}

Los datos sobre los componentes productivos de los huertos familiares, incluyendo tanto los componentes de vegetación y de origen animal, complejidad estructural, diversidad funcional y estrategias de gestión, las cuales apuntan a los sistemas de manejo de agricultura familiar en los huertos se obtuvieron de 100 hogares. Estos hogares fueron seleccionados mediante muestreo aleatorio para obtener datos representativos. Veinte hogares fueron seleccionados de cada una de las siguientes cinco comunidades (20 en cada comunidad, 100 en total): X-Maben ( $19^{\circ} 80^{\prime} \mathrm{N}$; 88 $88^{\circ} 17^{\prime} \mathrm{O}$; Tageo, 2016a), X-Pichil (19 68’ N; 88 42' O; Tageo, 2016b), X-Yatil (19 66' N; 88 44' O; Nuestro-México, 2016), San José II ( $\left.19^{\circ} 97^{\prime} \mathrm{N} ; 88^{\circ} 23^{\prime} \mathrm{N}\right)$ y Melchor Ocampo (200. $03^{\prime} \mathrm{N}$; $88^{\circ} 28^{\prime} \mathrm{O}$; Dices, 2016).

La recolección de datos consistió en una combinación de observaciones de campo cualitativas, encuestas cuantitativas y discusiones de grupos focales. Las encuestas cuantitativas se basaron en la colección de datos sobre la complejidad estructural de los huertos, la cual se puede entender como las dimensiones, o estratificación de los cultivos en los huertos y de su gestión vertical u horizontal para el aprovechamiento de las tierras; se condujo con base en observaciones de campo en conjunto con comentarios sobre la gestión y manejo de los huertos por parte de los integrantes de las familias. Las observaciones de campo fueron recogidas por los investigadores e involucraron hogares elegidos al azar, (debido a que un 100\% de los hogares en las comunidades cuentan con algún tipo de huerto casero, abordando desde unas cuantas plantas hasta un sistema complejo), con familias dispuestas a participar en la investigación. Estas observaciones de campo se utilizaron principalmente para fines de cotejar los datos cuantitativos tales como la complejidad estructural de los huertos familiares (vertical, horizontal, mixtos, manejo de suelo individual, comunal, mixtos, etc.) y su rendimiento (producción $\mathrm{m}^{-2}$, etc). 
Los grupos focales, o bien el conjunto de personas que participaron en encuestas y discusiones colectivas, corroboraron los datos recogidos a través de las observaciones de campo con información sobre conocimientos indígenas relacionados con la diversidad funcional y las estrategias de gestión utilizadas para el mantenimiento de sus propios huertos familiares. Los grupos focales fueron elegidos por dos razones: 1) permitir la comunicación entre los participantes para la colección de datos (Kitzinger, 1995); y 2) permitir una comprensión de las estrategias comunes de gestión y conocimientos con base en consensos comunales. Los grupos focales se utilizan para generar datos sobre el manejo sostenible de los recursos naturales, ya que el enfoque permite una mejor comprensión de cómo las comunidades administran sus propios recursos (Raymond et al., 2010; CBD, 2011; UNEP, 2012). Mediante los grupos focales se pudieron explorar cuestiones de bienestar social y relaciones dentro de la comunidad para entender la incidencia de los huertos familiares en las condiciones sociales positivas respecto al aumento del bienestar y los procesos de desarrollo a los que tienen acceso las familias. Los grupos focales fueron conformados por 15 a 25 miembros de ambos sexos, con la participación ocasional de niños. Esto se repitió en cada una de las cinco comunidades, lo que permitió una comprensión más representativa de las prácticas sociales y el conocimiento de los distintos niveles de producción de los huertos, la gestión y su manejo (estratificación de actividades con base en género y edad) así como las distintas estrategias para aprovechar la tierra con base en estructuras complejas (multifuncionalidad con base en diversificación de semillas, uso de tierra para crecer estructuras entrelazadas, etc.), que a su vez proporcionaron información sobre la función social de los huertos familiares a través de la construcción de la comunidad y redes. Los participantes provenían de hogares seleccionados al azar y se integraron voluntariamente. El líder de la comunidad también fue informado de los fines y objetivos de los grupos focales antes de la investigación.

La investigación implicó la participación de traductores (de maya a español) ya que los participantes en los grupos focales eran de origen maya, y su nivel de español era muy limitado. Los traductores consistieron en un grupo de estudiantes de la Universidad de Felipe Carrillo Puerto quienes son confiables tanto en español como en la lengua maya.

\section{RESULTADOS}

\section{Componentes de Producción y Complejidad Estructural}

Los resultados reportados fueron obtenidos en cinco comunidades indígenas en el estado de Quintana Roo. Algunos de los resultados pueden ser similares a resultados reportados por parte de otros autores que también han estudiado ambientes socio-culturales semejantes, lo que indica que la población Maya ha convertido los huertos caseros en herencia cultural. Generación tras generación, han diseñado y manejado huertos caseros multi-funcionales similares, para que a partir de ellos se puedan alcanzar los objetivos que se lleguen a plantear. Es por esto, que su herencia cultural resulta en datos afines.

Los datos recolectados sobre los componentes de producción, los diversos estratos y la diversidad vegetal de los huertos familiares, mostraron dos resultados clave. En primer lugar, se logró observar que existen en promedio de 4-5 estratos en cada uno de los sitios visitados ejemplo de esto se aprecia en la Figura 1 donde en el estrato inferior $0-1 \mathrm{~m}$ se da la mayor presencia de especie de índole ornamental, aromáticas, hortalizas y medicinales las cuales se ubican en la mayoría de los casos en la cercanía de los costados de la casa habitación; en el segundo estrato 1-2 m hay la presencia en su mayoría de gramíneas (maíz) y especies diversas de frutales en crecimiento; en el tercer estrato de 2-5 $\mathrm{m}$ se puede observar la presencia de especies como plátano, palmas y especies frutales entre otras, $y$ el estrato mayor de $5 \mathrm{~m}$ se compone de especies maduras de frutales, palmas y maderables, y una diversidad de plantas tanto herbáceas, arbustivas como leñosas (Cuadro 1). En segundo lugar, más del 95\% de los hogares estudiados tienen animales tanto domésticos como salvajes. El conjunto de estos componentes de la producción tanto vegetales como animales sirven a una variedad de propósitos, incluyendo la provisión de alimentos, forraje, medicinas y muchos otros (Cuadro 2).

Los huertos familiares también contienen especies de plantas estratificadas verticalmente, en cada estrato conteniendo plantas que pertenecen a una forma de vida específica. Esta tendencia se reconoce en huertos familiares de otras regiones (De Clerck y NegrerosCastillo, 2000). 


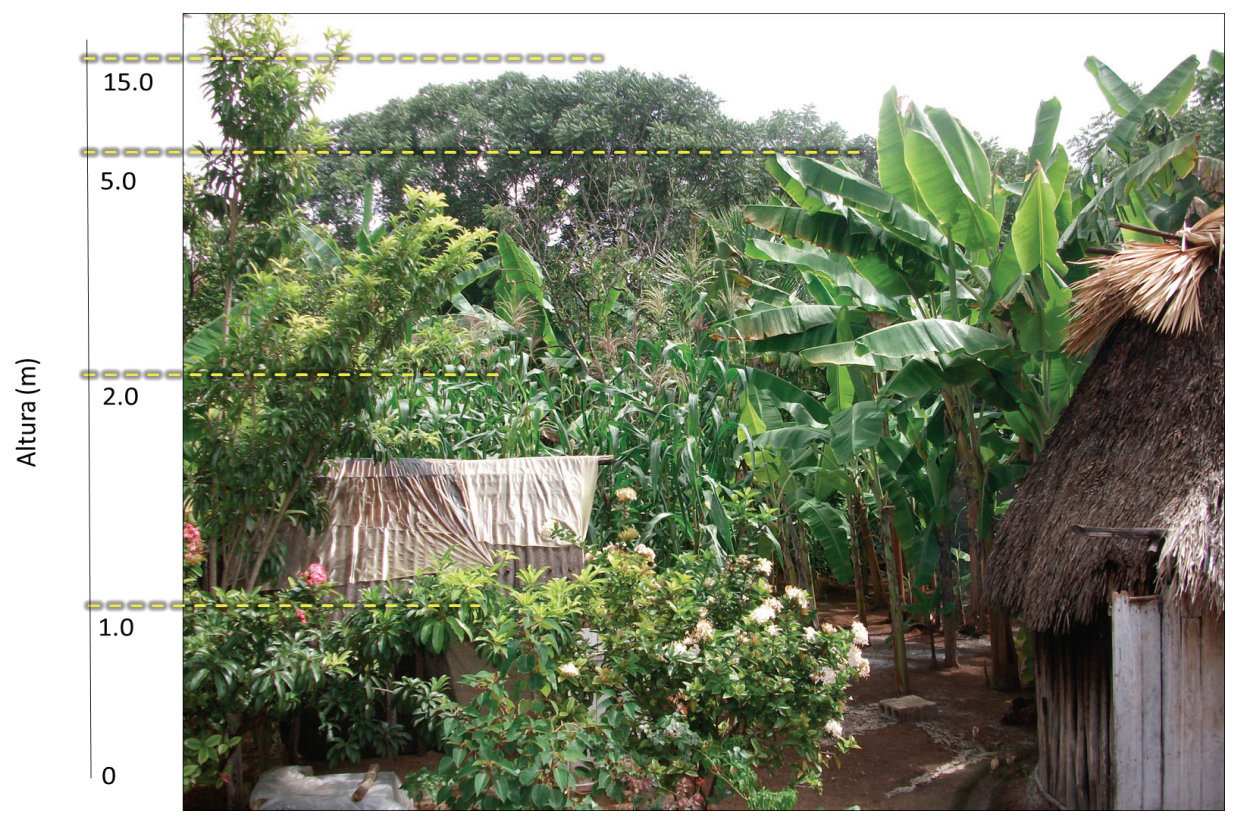

Figura 1. Estratos de un huerto casero en la comunidad de X-Maben, Quintana Roo.

Los hogares estudiados mostraron diversos focos de producción: algunos hogares se especializan en la producción animal (alrededor de $82 \%$, ya que los animales son la principal fuente de proteínas; cabras, borregos, puercos, gallinas y animales silvestres), mientras que otros en la medicina tradicional (alrededor de $90 \%$, con el fin de reducir el costo de la atención médica y proporcionar alivio inmediato) y otros más en la producción de alimentos (100\%, divididos entre hortalizas y árboles frutales) (Cuadro 3, Figura 2). Por lo tanto, los huertos familiares mayas consisten en interacciones complejas entre especies y están diversificados con estrategias de manejo flexibles y aportación de mano de obra externa mínima ya que la mayor parte de las actividades se realizan con mano de obra familiar (Caballero, 1992).

\section{Utilidades Obtenidas a Partir de los Huertos Familiares Tradicionales}

Uno de los resultados principales de las discusiones de los grupos focales fue el reconocimiento unánime del papel de la mujer en el manejo de los componentes productivos de los huertos familiares. Los hombres y otros miembros del hogar están a cargo de tareas de manejo, incluyendo la poda de los árboles, construcción y las ventas a pequeña escala para la creación de ingreso disponible. Esto sugiere que la estrategia de gestión de los huertos familiares es flexible y por lo general administrada dentro del hogar a través de mano de obra familiar.

En cuanto a los productos obtenidos de los huertos familiares en sí, la mayor parte de los productos alimenticios se emplean para el consumo familiar. Sólo una pequeña proporción del excedente es vendido, siendo compartido de vez en cuando con los vecinos y otros miembros de la comunidad. Esta es una tradición maya común mediante la cual se espera que los hogares compartan sus productos hortícolas para las festividades religiosas. Esto sirve para preservar la cultura, identidad y tradición a la vez que fomenta la cohesión social y la reproducción social. Las discusiones de los grupos focales sugieren que los miembros consideran que los variados servicios y funciones de los huertos familiares afectan a las comunidades de una manera positiva, por lo que es posible justificar la práctica de los huertos familiares como mucho más que sólo la necesidad de alimentos. Esta fue otra consideración importante discutida en los grupos focales.

El papel de los huertos familiares en la creación de redes sociales, cohesión y la construcción de las comunidades fue considerado importante durante las discusiones de los grupos focales. Los participantes destacaron la importancia de los huertos familiares en actividades cotidianas, así como un punto de partida en la creación de una buena relación entre las personas dentro de cada comunidad para relacionarse entre sí. Este es un hallazgo importante, ya que en las principales 
Cuadro 1. Especies de plantas más comunes encontradas en los huertos caseros de X-Maben, X-Pichil, X-Yatil, San José II y Melchor Ocampo en Felipe Carrillo Puerto, Quintana Roo.

\begin{tabular}{|c|c|c|c|}
\hline \multicolumn{4}{|c|}{ Organización vertical con el nombre regional y científico de la planta } \\
\hline \multicolumn{4}{|c|}{ Presencia por rangos de altura } \\
\hline $0-1$ & $0-2$ & $2-5$ & $5+$ \\
\hline$\ldots-\ldots-\ldots$ & $-------\ldots-$ & $---\mathrm{m}-\ldots-\ldots$ & $-\ldots-\ldots-\ldots-\ldots$ \\
\hline $\begin{array}{l}\text { Chile habanero } \\
\text { (Capsicum chinensis) }\end{array}$ & $\begin{array}{l}\text { Papaya } \\
\text { (Carica papaya) }\end{array}$ & $\begin{array}{l}\text { Plátano } \\
\text { (Musa sp.) }\end{array}$ & $\begin{array}{l}\text { Chique } \\
\text { (No identificado) }\end{array}$ \\
\hline $\begin{array}{l}\text { Calabaza } \\
\text { (Cucurbita mixta) }\end{array}$ & $\begin{array}{l}\text { Granada } \\
\text { (Punica granatum) }\end{array}$ & $\begin{array}{l}\text { Nance } \\
\text { (Byrsonima crassifolia) }\end{array}$ & $\begin{array}{l}\text { Coco } \\
\text { (Cocos nucifera) }\end{array}$ \\
\hline $\begin{array}{l}\text { Camote } \\
\text { (Ipomoea batatas) }\end{array}$ & $\begin{array}{l}\text { Tulipán } \\
\text { (Malvaviscus arboreus) }\end{array}$ & $\begin{array}{l}\text { Naranjo } \\
\text { (Citrus sinesis, C. Aurantium) }\end{array}$ & $\begin{array}{l}\text { Guarumbo } \\
\text { (Cecropia peltata } \sin . \text { C. obtusifolia) }\end{array}$ \\
\hline $\begin{array}{l}\text { Ruda } \\
\text { (Ruta chalpensis) }\end{array}$ & $\begin{array}{l}\text { Ortiga } \\
\text { (Urtica dioica) }\end{array}$ & $\begin{array}{l}\text { Guaje } \\
\text { (Leucaena leucocephala) }\end{array}$ & $\begin{array}{l}\text { Guano } \\
\text { (Sabal yapa, S. Mexicana) }\end{array}$ \\
\hline $\begin{array}{l}\text { Hierbabuena } \\
\text { (Mentha piperita) }\end{array}$ & $\begin{array}{l}\text { Dalia } \\
\text { (Dahlia sp.) }\end{array}$ & $\begin{array}{l}\text { Hoja santa } \\
\text { (Piper auritum) }\end{array}$ & $\begin{array}{l}\text { Cedro } \\
\text { (Cedrela odorata) }\end{array}$ \\
\hline $\begin{array}{l}\text { Epazote } \\
\text { (Chenopodium ambrosoides) }\end{array}$ & $\begin{array}{l}\text { Tabaco } \\
\text { (Nicotiana tabacum) }\end{array}$ & $\begin{array}{l}\text { Ciruelo } \\
\text { (Spondias mombin) }\end{array}$ & $\begin{array}{l}\text { Sabin } \\
\text { (No identificado) }\end{array}$ \\
\hline $\begin{array}{l}\text { Cilantro } \\
\text { (Coriandrum sativum) }\end{array}$ & $\begin{array}{l}\text { Maravilla } \\
\text { (Calendula officinalis L.) }\end{array}$ & $\begin{array}{l}\text { Chaya } \\
\text { (Cnidoscolus chayamansa) }\end{array}$ & $\begin{array}{l}\text { Kaska'at } \\
\text { (Luehea speciosa) }\end{array}$ \\
\hline $\begin{array}{l}\text { Rosa } \\
(\text { Rosa gallica })\end{array}$ & $\begin{array}{l}\text { Caña de azúcar } \\
\text { (Saccharum officinarum) }\end{array}$ & $\begin{array}{l}\text { Buganvilia } \\
\text { (Bougainvillea glabra) }\end{array}$ & $\begin{array}{l}\text { Pinch } \\
\text { (No identificado) }\end{array}$ \\
\hline $\begin{array}{l}\text { Frijol } \\
\text { (Phaseolus sp.) }\end{array}$ & $\begin{array}{l}\text { Maíz } \\
\text { (Zea mays) }\end{array}$ & $\begin{array}{l}\text { Xiyiat } \\
\text { (No identificada) }\end{array}$ & $\begin{array}{l}\text { Anona } \\
\text { (Annona squamosa, A. reticulata) }\end{array}$ \\
\hline \multirow[t]{4}{*}{$\begin{array}{l}\text { Albahaca } \\
(\text { Ocimum bailicum })\end{array}$} & $\begin{array}{l}\text { Ave del paraíso } \\
\text { (Strelitzia reginae) }\end{array}$ & $\begin{array}{l}\text { Mandarina } \\
\text { (Citrus reticulata) }\end{array}$ & $\begin{array}{l}\text { Mango } \\
\text { (Mangifera sp.) }\end{array}$ \\
\hline & & $\begin{array}{l}\text { Huele de noche } \\
\text { (Cestrum nocturnum L.) }\end{array}$ & $\begin{array}{l}\text { Zapote mamey } \\
\text { (Pouteria sapota, P. mammosa) }\end{array}$ \\
\hline & & $\begin{array}{l}\text { Palma } \\
\text { (Veitchia merillii) }\end{array}$ & $\begin{array}{l}\text { Ramón } \\
\text { (Brosimum alicastrum) }\end{array}$ \\
\hline & & & $\begin{array}{l}\text { Aguacate } \\
\text { (Persea americana) }\end{array}$ \\
\hline
\end{tabular}

agendas de política internacional tales como: Agenda 21, Objetivos de Desarrollo del Milenio y más recientemente Objetivos del Desarrollo Sostenible, impulsadas por la Organización de Naciones Unidas, se deja de lado las diferentes formas a través de las cuales puede construirse cohesión social a partir de huertos familiares. Aunque hay un reconocimiento del intercambio de ideas y conocimientos tradicionales que se está llevando acabo, nociones como la soberanía alimentaria, identidad, relación y construcción de las comunidades a menudo se consideran el resultado de la acumulación positiva de la seguridad alimentaria y económica, en lugar de una consecuencia paralela de la práctica de los huertos familiares.
En este sentido, los grupos focales son conscientes de los usos y funciones de los huertos familiares los cuales pueden ser considerados bajo el espectro de la seguridad, incluyendo la financiera, nutrimental, social y sanitaria; destacando para ellos los siguientes: (1) alimentos o víveres; (2) medicinas (para los humanos y animales domésticos); (3) forrajes; (4) aromáticos (condimentos, perfumes, etc.); (5) edulcorantes; (6) bebidas alcohólicas o no alcohólicas; (7) especias; (8) estimulantes; (9) ceremoniales (amuletos, magia, rituales); (10) drogas (alucinógenos, narcóticos, tranquilizantes); (11) resinas; (12) miel; (13) aceite (comestible e industrial); (14) cercas; (15) rompevientos; (16) herramientas para la agricultura, 
Cuadro 2. Algunas de las plantas y animales encontrados en los huertos familiares de las comunidades: X-Maben, X-Pichil, X-Yatil, San José II y Melchor Ocampo en Felipe Carrillo, Quintana Roo, México, y sus usos.

Especies vegetales

\begin{tabular}{|c|c|c|c|}
\hline Nombre Local & Nombre Científico & Familia & Usos \\
\hline Chincuya & Annona purpurea & Anonaceae & $\begin{array}{l}\text { Alimentos, aromático, artesanías, construcción de viviendas, leña } \\
\text { y madera }\end{array}$ \\
\hline Achiote & Bixa orellana & Bixaceae & $\begin{array}{l}\text { Alimentos, aromático, ceremonial, colorantes, condimentos, } \\
\text { industrial, leña y medicinal }\end{array}$ \\
\hline Chaka & Bursera simarouba & Burseraceae & $\begin{array}{l}\text { Artesanías, seto vivo, ceremonial, sujeción de suelo, instrumentos, } \\
\text { leña, madera, medicinal, taninos }\end{array}$ \\
\hline Nance & Byrsonima crassifolia & Malpighiaceae & $\begin{array}{l}\text { Alimentos, artesanías, ceremonial, colorantes, construcción, } \\
\text { forraje, sujeción de suelo, leña, madera, medicinales, ornamentales }\end{array}$ \\
\hline Papaya & Carica papaya & Caricaceae & Alimentos, bebidas, industrial, medicinales, ornamentales \\
\hline Cedro & Cedrela odorata & Meliaceae & $\begin{array}{l}\text { Artesanías, madera, sujeción de suelo, leña, repelentes, } \\
\text { ornamentales }\end{array}$ \\
\hline Limón dulce & Citrus limonia & Rutaceae & $\begin{array}{l}\text { Alimentos, aromático, bebidas, condimentos, leña, medicinales, } \\
\text { ornamentales }\end{array}$ \\
\hline Pajarito & Cordia alliodora & Boraginaceae & Artesanías, instrumentos, leña, madera, medicinales, ornamentales \\
\hline Jícara & Crescentia cujete & Bignoniaceae & $\begin{array}{l}\text { Alimentos, material de construcción, ceremonial, utensilio } \\
\text { doméstico, médico, producción de miel }\end{array}$ \\
\hline Cocoíte & Gliricidia sepium & Fabaceae & $\begin{array}{l}\text { Forraje, artesanías, seto vivo, colorantes, fijación de nitrógeno, } \\
\text { leña, medicinales, repelentes, sombra, taninos, ornamentales }\end{array}$ \\
\hline Aguacate & Persea americana & Lauraceae & $\begin{array}{l}\text { Alimentos, cosméticos, condimentos, industriales, medicinales, } \\
\text { madera }\end{array}$ \\
\hline
\end{tabular}

Especies animales

\begin{tabular}{|c|c|c|}
\hline Nombre local & Nombre Científico & Categorías de uso en los hogares \\
\hline Perro & Cannis familiaris & Protección, mascota \\
\hline Pollo & Gallus gallus & Comida, venta \\
\hline Puerco & Sus scrofa & Comida, venta \\
\hline Pavo & Melleagris gallopavo & Comida, venta \\
\hline Pato & Anas sp. & Comida, venta \\
\hline Borrego & Ovis aries & Comida, venta \\
\hline Gato & Felis silvestris catus & Protección, mascota \\
\hline Caballo & Equus caballus & Trabajo \\
\hline Ganado & Bos indicus, B.taurus & Comida, trabajo \\
\hline Burro & Equus asinus & Trabajo \\
\hline Conejo & Sylvilagus sp. & Comida, venta, mascota \\
\hline Mula & E. caballu $x$ asinus & Trabajo \\
\hline Ganso & Anser anser & Protección, ornamental \\
\hline Ave de corral & Numida meleagris & Ornamental \\
\hline Aquijones & Melipona sp. & Miel como alimento y medicina \\
\hline
\end{tabular}


la caza y la pesca; (17) fibras (textiles, cuerdas y cestería); (18) construcción (muebles o casas); (19) artesanías; (20) instrumentos musicales; (21) ceras; (22) colorantes; (23) control biológico (insecticidas, fungicidas, herbicidas); (24) cosmético; (25) uso doméstico (cocina, embalaje, adhesivos de secado, etc.); (26) bioenergía (carbón, leña, aceite); (27) control de la erosión del suelo; (28) caucho y látex; (29) ornamental o estético; (30) taninos; (31) tóxico (venenoso para el hombre y los animales domésticos); (32) picaduras de abejas con fines médicos; y (33) abono verde.

\section{Servicios Funcionales de los Huertos Familiares}

Tomando como base los criterios de la clasificación cuádruple de la Evaluación de los Ecosistemas del Milenio 2001 (MEA, 2005), los grupos focales además de identificar los diversos usos de los productos cultivados, también lograron vislumbrar una serie de otros servicios de los huertos familiares como unidades en sí. Estos incluirían (1) servicios de provisión: productos obtenidos del sistema, (2) servicios de regulación: los beneficios de la regulación del sistema incluyen la mejora de la calidad del aire, la regulación del clima y la disminución de la susceptibilidad a los desastres naturales, (3) servicios culturales: servicios no materiales recolectados del enriquecimiento espiritual, estatus social, la recreación, el entretenimiento, la atención plena, relación social y las redes sociales, y (4) servicios de apoyo: servicios considerados importantes para otras funciones de los ecosistemas, tales como la conservación del suelo, la fotosíntesis y ciclos de nutrientes.

\section{DISCUSIÓN}

Los huertos familiares son sistemas complejos. Son estrategias resilientes probadas con el tiempo (Galhena et al., 2013) y consisten en estrategias de gestión flexibles a nivel de hogar (Caballero, 1992). Los huertos familiares estudiados son un tipo muy específico (Lope-Alzina y Howard, 2012), se debe a que los huertos familiares de comunidades indígenas incluyen un gran número de especies de plantas silvestres y cultivadas que se estructuran en diferentes capas verticales y que son manejados con el objetivo de transmitir conocimientos de una manera inter-generacional. Por otra parte (Leclerc y Thuillet, 2014), la combinación intrica de especies de plantas está dispuesta horizontalmente lo que toma en consideración a los tipos de suelo específicos y a los ciclos de nutrientes para la mejor producción durante todo el año. Lo que hace que la combinación de estos factores sea tan sorprendente en el contexto maya es que

Cuadro 3. Número de especies de plantas útiles, por categoría, presentes en los huertos caseros de las comunidades de X-Maben, X-Pichil, X-Yatil, San José II y Melchor Ocampo, en el municipio de Felipe Carrillo Puerto.

\begin{tabular}{|c|c|c|c|c|c|c|c|c|c|c|}
\hline \multirow{2}{*}{ Categoría de uso } & \multicolumn{2}{|c|}{ X-Maben } & \multicolumn{2}{|c|}{ X-Pichil } & \multicolumn{2}{|c|}{ X-Yatil } & \multicolumn{2}{|c|}{ San José II } & \multicolumn{2}{|c|}{ Melchor Ocampo } \\
\hline & No. & $\%$ & No. & $\%$ & No. & $\%$ & No. & $\%$ & No. & $\%$ \\
\hline Medicinas & 78 & 28.5 & 59 & 23.9 & 108 & 34.6 & 144 & 41.0 & 96 & 35.5 \\
\hline Alimentos & 99 & 36.2 & 86 & 34.9 & 94 & 30.1 & 102 & 29.0 & 71 & 26.3 \\
\hline Construcción & 9 & 3.3 & 13 & 5.3 & 21 & 6.7 & 18 & 5.12 & 11 & 4.0 \\
\hline Artesanías & 8 & 2.9 & 14 & 5.7 & 9 & 2.9 & 15 & 4.3 & 8 & 3.0 \\
\hline Ornamentales & 13 & 4.76 & 16 & 6.5 & 11 & 3.5 & 10 & 2.8 & 9 & 3.3 \\
\hline Maderas & 9 & 3.3 & 11 & 4.4 & 10 & 3.2 & 15 & 4.3 & 12 & 4.4 \\
\hline Forrajes & 19 & 6.9 & 12 & 4.8 & 13 & 4.2 & 10 & 2.8 & 15 & 5.5 \\
\hline Herramientas & 9 & 3.3 & 7 & 2.8 & 11 & 3.5 & 8 & 2.2 & 10 & 3.7 \\
\hline Combustibles & 18 & 6.6 & 19 & 7.7 & 22 & 7.0 & 19 & 5.4 & 23 & 8.5 \\
\hline Bienes domésticos & 11 & 4.0 & 9 & 3.6 & 13 & 4.2 & 10 & 2.8 & 15 & 5.5 \\
\hline Total & 273 & 99.76 & 246 & 100 & 312 & 99.9 & 351 & 99.72 & 270 & 99.7 \\
\hline
\end{tabular}




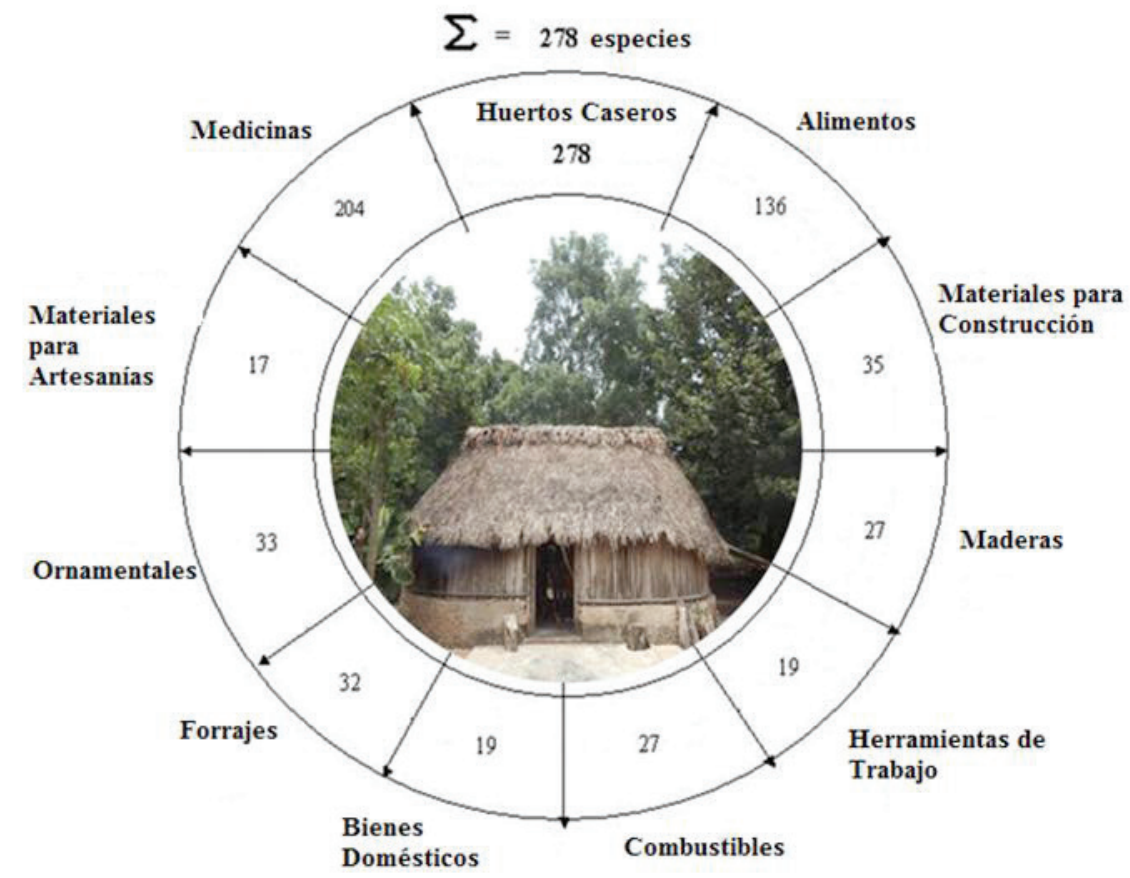

Figura 2. Número de especies y productos múltiples que se pueden obtener de los huertos tradicionales Mayas de las comunidades estudiadas en el municipio de Felipe Carrillo Puerto, Quintana Roo (Toledo, 2003).

las comunidades no consideran estos aspectos como unidades separadas de análisis, sino como un conjunto, donde los factores políticos, económicos, culturales y sociales están vinculados entre sí y relacionados con los factores biológicos, agrícolas y ecológicos. Leclerc y Thuillet (2014) observaron patrones similares de agricultura familiar en diferentes partes del mundo.

Aunque los grupos focales se están volviendo cada vez más importantes en el estudio de puntos de vista sociales, al usarlos se descartan las percepciones individuales, priorizando las comunales. La problemática de los grupos focales es que ciertas voces y discursos podrían ser ignorados, ya que no todos los miembros se sienten cómodos hablando en un ambiente grupal. Tal vez podría incluso haber jerarquías locales que los investigadores desconocen, incluyendo jerarquías basadas en género o condición social. Para evitar esto, la investigación podría haber sido llevada a cabo por medio de entrevistas individuales semiestructuradas. Otra alternativa podría ser la división de los grupos focales basada en género. Esto habría permitido una mejor comprensión de los huertos familiares como un espacio para el bienestar social, pero además, y aún más importante, habría producido datos desglosados por género sobre las prácticas de manejo. Esto es importante cuando se considera el hecho de que los huertos familiares, en la mayoría de los casos, suelen ser administrados por la mujer jefa de familia (Caballero, 1992). Debido a esto, se puede argumentar que las mujeres están más equipadas y preparadas en los temas del manejo y la diversidad funcional. También pasan más tiempo en los huertos familiares en comparación con los hombres, y tienden a ser las encargadas de decidir cómo utilizar los productos para el consumo del hogar (Montagnini, 2006). No sólo esto, sino una perspectiva de género también habría podido arrojar luz sobre las formas en que las subsecciones de la sociedad se relacionan entre sí. Por ejemplo, las relaciones de las mujeres, el intercambio de conocimientos y el bienestar están más intrínsecamente relacionados con los huertos familiares que las relaciones de los hombres, en parte porque estos también tienen sus espacios de trabajo para discutir entre ellos.

Aparte de la adición de una comprensión de género de las prácticas de los huertos familiares y su importancia social, un muestreo selectivo en lugar de uno aleatorio podría haber mejorado el estudio. 
Dada la naturaleza aleatoria del estudio, se puede argumentar que fueron ignorados ciertos aspectos de los huertos familiares. Estos incluyen la proximidad a otros espacios sociales, caminos y ciudades, que podrían tener un efecto sobre las prácticas, opiniones y relaciones de los huertos familiares.

Por último, es importante reconocer las limitaciones del uso de traductores. Aunque en este caso, hubo poca o ninguna alternativa, los traductores tienen sesgos incrustados en su propia comprensión de las comunidades y los participantes involucrados. Esto probablemente influyó en las traducciones de las discusiones de los grupos focales. Por otra parte, para los participantes el uso de estos traductores podría haber tenido consecuencias pasadas por alto por los investigadores. Estas incluyen cuestiones tales como querer verse de cierta manera ante los traductores y por lo tanto cambiar sus respuestas. A pesar de que no había alternativa, es crucial tener en cuenta estas limitaciones potenciales al considerar los resultados y conclusiones.

La diversidad de las funciones de los huertos familiares revela tres principales hallazgos. En primer lugar, los huertos familiares juegan un papel importante en la creación de seguridad económica y alimentaria, que a su vez facilita la seguridad de subsistencia. En segundo lugar, los huertos familiares tienen una presencia e influencia en las relaciones y actividades del día a día a niveles de hogar, fraternales y de comunidad. En tercer lugar, dentro de la multiplicidad de las funciones de los huertos familiares, es posible ver que apoyan la creación y recreación de los dos ecosistemas para la producción de alimentos, así como las relaciones sociales de manera sostenible e interrelacionada.

Teniendo en cuenta que la seguridad alimentaria abarca las nociones de la disponibilidad, la utilización de la accesibilidad y la estabilidad, es posible entender las formas en que esta investigación corrobora el discurso dominante sobre los huertos familiares: que la principal y más importante razón para la práctica y el mantenimiento de huertos familiares es para la producción continua de las variadas fuentes de alimentos para el consumo a nivel del hogar (FAO, 1996; Caballero, 1992).

Además del reconocimiento de los huertos familiares como fuente de alimento, es importante tener en cuenta también sus funciones sociales. Los huertos familiares representan un ejemplo de desarrollo desde abajo hacia arriba, porque las comunidades iniciaron la práctica por sí mismas. Esto implica que los huertos familiares son una práctica y un enfoque que permite que las comunidades y actores locales expresen sus inquietudes y conocimientos para definir las vías de desarrollo (Galhena et al., 2013). Ejemplo de ello es la participación activa en el manejo de los huertos familiares por los diferentes miembros de la familia. Las discusiones de los grupos focales acerca de los diferentes métodos de gestión demostraron cómo los huertos familiares son clave para permitir que las comunidades se conviertan en agentes de su propio cambio. Las comunidades tienen control sobre su seguridad alimentaria, económica, de medios de vida y social, y son flexibles y adaptables a las condiciones cambiantes. El papel de los huertos familiares es diferente dependiendo de si se analizan de manera holística o por medio de un análisis de sus diversos componentes y funciones. El huerto familiar, como unidad, tiene importantes significados sociales y su uso simbólico es esencial en la creación de conversaciones, relaciones y nociones compartidas de identidad. Al tener en cuenta los cultivos de forma individual, por el contrario, uno de los papeles claves de los huertos familiares es la producción de cultivos para mitigar las desigualdades sociales y la pobreza, proporcionando alimentos, medicinas y ornamentos. Los huertos familiares pueden clasificarse no sólo en términos de suelo, productos, tamaño y rendimiento, sino también en cuanto a los métodos de gestión utilizados. Esto a su vez hace hincapié en los casos de la identidad y la diversidad de los huertos familiares en las diferentes regiones del mundo.

El hecho de que los huertos familiares y sus propósitos y funciones se pueden entender de esta manera sugiere que los huertos familiares juegan un papel en las realidades y vidas indígenas en diversas formas. Esta es una consideración clave, debido a que en el estudio de los huertos familiares, también se está considerando una serie de estilos de vida y realidades. Sin embargo, más importante aún, la consideración de las diversas funciones de los huertos familiares define a las comunidades indígenas como agentes de su propio bienestar y seguridad.

También es importante tener en cuenta las limitaciones en las prácticas de los huertos familiares en el contexto maya. En primer lugar, no existe un organismo como una cooperativa al que las comunidades puedan vender sus excedentes de producción. Para ello, tal vez el establecimiento de 
una asociación o un organismo comunitario puede mejorar el acceso a los mercados y otras instituciones públicas, para reducir los costos asociados con la venta de sus productos. Esto también podría facilitar el hallazgo de nuevas oportunidades de demanda, así como para obtener la formación y el conocimiento y la experiencia técnica de organismos externos tales como agencias gubernamentales, que a su vez podrían ayudar a aumentar el rendimiento y la producción. Las cooperativas también podrían mejorar la relación y comunicación entre las comunidades y el Estado, mitigando una serie de problemas en las comunidades que no están necesariamente asociados con los huertos familiares, como la infraestructura, el intercambio de información y la modernización de la zona a través de nuevos métodos y empresas, convirtiéndose en actividades mercantiles que las permitan insertarse en la economía global. Mientras que al hacer esto se podría perder la esencia de lo "familiar" de los huertos caseros estudiados, lo que se propone es una sinergia entre los modos de producción en los huertos conjunto los sistemas de demanda y oferta en las comunidades. El propósito con esto no sería el de maximizar las economías de escala, si no el de utilizar los mercados disponibles como fuentes de ingresos modernos para mejorar el manejo de los huertos caseros. Cabe mencionar asimismo, que en la escuela del desarrollo incluyente, se habla del potencial de los productores de huertos familiares al vender su exceso en los mercados para de un lado lograr generar ingresos adicionales y por otro generar seguridad alimentaria (Gupta, 2014).

Por último, es importante recordar que los huertos familiares permiten la flexibilidad, cultura, identidad y resiliencia. En este sentido, los organismos gubernamentales deben considerar los diferentes tipos de prácticas de agricultura familiar involucradas en el manejo de los huertos familiares para crear políticas que se ajusten a las realidades multidimensionales de las experiencias indígenas y al mismo tiempo que ayuden al desarrollo económico, comercial y público.

\section{CONCLUSIONES}

Los huertos familiares juegan un papel crucial para las comunidades mayas de: X-Maben, X-Pichil, X-Yatil, San José II y Melchor Ocampo en el estado de
Quintana Roo, debido a que sirven para proporcionar seguridad alimentaria, económica y social. Esto es porque ofrecen una diversidad de cultivos, altos rendimientos y producción durante todo el año, pero también implican un espacio flexible para la producción de alimentos nutritivos y variados. De hecho, consisten de una gran diversidad de especies, estructuras complejas, una aportación externa mínima y sistemas de manejo flexible para combinar componentes agrícolas, forestales y animales. Los huertos familiares también sirven a las comunidades por medio de la atención de la medicina tradicional con base en plantas que ayudan a afrontar crisis imprevistas, así como un alivio eficiente en costos y autosuficiente inmediato. Tal vez más destacable, la investigación muestra que los huertos familiares permiten la resiliencia social y construcción de comunidades a través de la provisión de espacios para el intercambio de conocimientos y bienes. Esto, a su vez, permite la modernización y rápida diversificación de comunidades aisladas que tiene implicaciones para los enfoques rurales desde abajo hacia arriba para el desarrollo. La investigación también muestra que, la mayoría de las actividades diarias, personales y comunales, tienen lugar alrededor del huerto familiar, lo que le permite desempeñar el papel de una entidad y espacio para el desarrollo. En este sentido, es posible argumentar que los huertos familiares tocan cada una de las tres esferas de desarrollo reconocidas: social, económica y ambiental, lo que sugiere que la literatura sobre los huertos familiares, por lo tanto, necesita considerar una comprensión más precisa del papel que desempeñan a nivel popular, donde las comunidades y las personas utilizan los huertos familiares para empoderarse a sí mismos como conductores y agentes de su propio cambio.

\section{AGRADECIMIENTOS}

Queremos manifestar nuestro agradecimiento a las comunidades mayas del estado de Quintana Roo, México, por compartir con nosotros su tiempo y conocimientos. Así también nuestro agradecimiento para Blanca Dianeli Tuz Bacab, Laura Elena Chuc Castro y Sulmy Argeli Coh Tuk por su gentil acompañamiento, y apoyo como traductoras mayaespañol durante el presente estudio. 


\section{LITERATURA CITADA}

Angel-Pérez, A. L. 2013. Homegardens and the dynamics of Totonac domestic groups in Veracruz, Mexico. Anthropological Notebooks 19: 5-22.

Arias Reyes, L. M. 2012. El huerto familiar o solar maya-yucateco actual. pp. 111-130. In: R. Mariaca Méndez (ed.). El huerto familiar del sureste de México. ECOSUR. México.

Bautista, F., D. Palma-López y W. Huchin-Malta. 2005. Actualización de la clasificación de los suelos del Estado de Yucatán. pp. 105-122. In: F. Bautista y G. Palacio (eds.). Caracterización y manejo de los suelos de la Península de Yucatán: Implicaciones agropecuarias, forestales y ambientales. Universidad Autónoma de Campeche, Universidad Autónoma de Yucatán, Instituto Nacional de Ecología. México, D. F. ISBN: 968-5722-13-7.

Brownrigg, L. A. 1985. Home gardening in international development: What the literature shows, including an an annotated bibliography, and inventories of International Organizations involved in home gardening and their projects. The League for International Food Education. Washington, DC, USA.

Caballero, J. 1992. Maya homegardens: Past, present and future. Etnoecológica 1: 35-54.

Cahuich-Campos, D. 2012. El huerto maya y la alimentación cotidiana de las familias campesinas de X-Mejía, Hopelchén, Campeche. pp. 197-229. In: R. Mariaca Méndez (ed.). El huerto familiar del sureste de México. ECOSUR. México.

Cámara-Córdova, J. 2012. Contribución del huerto familiar a la economía rural, a la adaptación al cambio climática y a la conversión productiva en Tabasco, México. pp. 372-390. In: R. Mariaca Méndez (ed.). El huerto familiar del sureste de México. ECOSUR. México.

CBD (Convention of Biological Diversity). 2011. The Tkarihwaié:ri code of ethical conduct to ensure respect for the cultural and intellectual heritage of indigenous and local communities relevant to the conservation and sustainable use of biological diversity. http://www.cbd.int/traditional/code/ethicalconductbrochure-en.pdf (Consulta: agosto 15, 2015).

CONEVAL (Consejo Nacional de Evaluación de la Política de Desarrollo Social). 2013. Informe de pobreza en México, 2012. CONEVAL. México, D. F.

DBCity (Dainik Bhaskar Group). 2016. Felipe Carrillo Puerto. http://es.db-city.com/M\%C3\%A9xico-Quintana-Roo-FelipeCarrillo-Puerto (Consulta: septiembre 18, 2016).

De Clerck, F. A. J. and P. Negreros-Castillo. 2000. Plant species of traditional Mayan homegardens of Mexico as analogs for multistrata agroforests. Agrofor. Syst. 48: 303-317.

Dices (Directorio Cartográfico de España y Latinoamerica). 2016. Melchor Ocampo, Quintana Roo, Felipe Carrillo Puerto, Chetumal. http://www.dices.net/mapas/mexico/mapa. php?nombre=Melchor-Ocampo\&id=110414 (Consulta: octubre 29, 2016).

Eyzaguirre, P. B. and O. F. Linares. 2004. Home gardens and agrobiodiversity. Smithsonian Institution Press. Washington, DC, USA.

FAO (Food and Agriculture Organization). 1996. World Food Summit. Disponible en: http://www.fao.org/wfs/ (Consulta: agosto 8, 2015).
FAO (Food and Agriculture Organization). 2013. State of food and agriculture. Food system for better nutrition. FAO. Rome, Italy. ISBN 978-92-5-107671-2.

Fernandes, E. C. M. and P. K. R. Nair. 1986. An evaluation of the structure and function of tropical homegardens. Agric. Syst. 21: $279-310$

Flores Guido, J. S. 2012. Diversidad florística, usos y origen de material genético de las especies de los huertos familiares de la Península de Yucatán. pp. 149-175. In: R. Mariaca Méndez (ed.). El huerto familiar del sureste de México. ECOSUR. México.

Galhena, D. H, R. Freed, and K. M. Maredia. 2013. Home gardens: A promising approach to enhance household food security and wellbeing. Agric. Food Sec. 2: 8. DOI: 10.1186/2048-7010$2-8$

Gupta, J., I. Baud, R. Bekkers, S. Bernstein, I. Boas, V. Cornelissen, M. Iguchi, N. Kanie, R. E. Kim, M. Bastos Lima, P. Obani, P. Schoof, C. Stevens, and D. van Zoomeren. 2014. Sustainable Development Goals and Inclusive Development. POST2015/ UNU-IAS Policy Brief \#5. United Nations University Institute for the Advanced Study of Sustainability. Tokyo.

INEGI (Instituto Nacional de Estadística y Geografía). 2015. Disponible en: http://sipse.com/novedades/quintana-roopoblacion-indigena-encuesta-intercensal-2015-inegi-lenguamaya-184159.html (Consulta: septiembre 18, 2016).

INEGI (Instituto Nacional de Estadística y Geografía). 2016. http://www.cuentame.inegi.org.mx/monografias/informacion/ qroo/poblacion/diversidad.aspx tema $=$ me $\& \mathrm{e}=23$ (Consulta: septiembre 18, 2016).

Kitzinger, J. 1995. Qualitative research. Introducing focus groups. BMJ 311: 299-302.

Kumar, B. M. and P. K. R. Nair. 2004. The enigma of tropical homegardens. Agrofor. Syst. 61: 135-152.

Leclerc, C. and A. C. Thuillet. 2014. Wild and cultivated biodiversity and natural resource management. Montpellier, France. Agrop. Int. 19: 35-47.

Lope-Alzina, D. G. and P. L. Howard. 2012. The structure, composition and functions of homegardens: Focus on the Yucatán Peninsula. Etnoecológica 9: 17-41.

Mariaca M., R. 2012. El huerto familiar del sureste de México. ECOSUR. México, D. F.

MEA (Millennium Ecosystem Assessment). 2005. Ecosystems and human well-being: Synthesis. Island Press. Washington, DC, USA.

Méndez, V. E., R. Lok, and E. Somarriba. 2001. Interdisciplinary analysis of homegardens in Nicaragua: Micro-zonation, plant use and socioeconomic importance. Agrofor. Syst. 51: 85-96.

Mohan, S., J. R. R. Alavalapati, and P. K. R. Nair. 2006. Financial analysis of homegardens: A case study from Kerala state, India. pp. 283-298. In: B. M. Kumar and P. K. R. Nair (eds.). Tropical homegardens: A time-tested example of sustainable agroforestry. Advances in Agroforesetry. Volume 3. Springer. The Netherlands.

Montagnini, F. 2006. Homegardenns of Mesoamerica: Biodiversity, food security, and nutrient management. pp. 61-84. In: B. M. Kumar and P. K. R. Nair (eds.). Tropical homegardens: A time-tested example of sustainable agroforestry. Advances in Agroforesetry. Volume 3. Springer. The Netherlands. 
PNUD (Programa de las Naciones Unidas para el Desarrollo). 2016. Desarrollo inclusivo. http://www.undp.org/content/ undp/en/home/ourwork/povertyreduction/focus_areas/focus inclusive_development.html (Consulta: octubre 28,2016 ).

Raymond, C. M., I. Fazey, M. S. Redd, L. C. Stringer, G. M. Robinson, and A. C. Evely. 2010. Integrating local and scientific knowledge for environmental management. J. Environ. Manage. 91: 1766-1777.

Rebollar-Domínguez, S., V. J. Santos-Jiménez, N. A. TapiaTorres y C. P. Pérez-Olvera. 2008. Huertos familiares, una experiencia en Chancah Veracruz, Quintana Roo. Polibotánica 25: $135-154$.

Soemarwoto, O. 1987. Homegardens: A traditional agroforestry system with a promising future. pp: 157-170. In: H. A. Steppler and P. K. R. Nair (eds.). Agroforestry: A decade of development. ICRAF. Nairobi, Kenya.

Tageo. 2016a. Melchor Ocampo, Quintana Roo. http://www.tageo. com/index-e-mx-v-23-d-m2398966.htm (Consulta: octubre 28, 2016).

Tageo. 2016b. X-Pichil, Quintana Roo. http://www.tageo.com/ index-e-mx-v-23-d-m2399119.htm (Consulta: octubre 28, 2016).
Toledo, V. M. 2003. Los pueblos indígenas, actores estratégicos para el Corredor Biológico Mesoamericano. Biodiversitas 47: $8-15$.

Torquebiau, E. 1992. Are tropical agroforestry home gardens sustainable? Agric. Ecosyst. Environ. Elsevier. 41: 189-207.

Torquebiau, E. and E. Penot. 2006. Ecology versus economics in tropical multistrata agroforests. pp. 269-282. In: B. M. Kumar and P. K. R. Nair (eds.). Tropical homegardens: A time-tested example of sustainable agroforestry. Advances in Agroforesetry. Volume 3. Springer. The Netherlands.

UNEP (United Nations Environment Programme). 2012. Consideration of initial elements: recognizing indigenous and local knowledge and buildign synergies with science. IPBES/1/INF/5.http://www.ipbes.net/plenary/ipbes-1.html (Consulta: diciembre 4, 2015).

United Nations. 2000. Millennium Development Goals. http:// www.un.org/millenniumgoals/poverty.shtml (Consulta: agosto 25, 2015).

Vía Campesina. 1996. World Food Summit. https://viacampesina. org/en/index.php/organisation-mainmenu-44 (Consulta: 18 septiembre 18, 2016).

Wiersum, K. F. 1982. Tree gardening and taungya in Java: Examples of agroforestry techniques in the humid tropics. Agrofor. Syst. 1: $53-70$. 